\section{Effectiveness of Colchicine Therapy in 4 Cases of Retroperitoneal Fibrosis Associated with Autoinflammatory Diseases}

\section{To the Editor:}

Retroperitoneal fibrosis (RPF) is a fibroinflammatory process affecting the retroperitoneal structures. There are no guidelines for its diagnosis or therapy. We describe 4 cases of RPF associated with autoinflammatory diseases, in which treatment with colchicine allowed RPF regression.

RPF is an uncommon disease, with unclear pathogenesis. Systemic manifestations (fever, anorexia, weight loss), often associated with local symptoms (flank pain, leg edema, abdominal discomfort), are related to the entrapment of retroperitoneal structures by the fibrotic plaques. Initial therapy aims at restoring the function of the affected organs with the application of ureteral stents, followed by immunosuppressive therapy. Sometimes, ureteral replacement or kidney autotransplant may be necessary ${ }^{1}$. Colchicine, a tricyclic alkaloid, interferes with microtubule formation, altering mitosis and inhibiting many steps in the inflammatory process. It reduces adhesion of neutrophils to endothelium, inhibiting neutrophil migration, and blocks the in vitro release of fibronectin from alveolar macrophages ${ }^{2,3}$. It is approved by the US Food and Drug Administration for the treatment and prophylaxis of gout flares, but is used in the treatment of familial Mediterranean fever (FMF), recurrent pericarditis, primary biliary cirrhosis, Behçet's disease, relapsing polychondritis, Sweet's syndrome, scleroderma, amyloidosis, leukocytoclastic vasculitis, epidermolysis bullosa ${ }^{4}$, and idiopathic pulmonary fibrosis ${ }^{5}$.

Case 1. A 48-year-old man with recurrent fevers since childhood, peritonitic abdominal pain since 1998, and periaortic RPF since 2004 (Figure $1)$, under home therapy with methylprednisolone (4 mg/day), cyclophosphamide $(1 \mathrm{mg} / \mathrm{kg} /$ day $)$ and tamoxifen $(10 \mathrm{mg} /$ day $)$, came to our attention in 2006. His history led us to hypothesize FMF. Corticosteroids, cyclophosphamide, and tamoxifen were suspended and colchicine therapy was started at a dosage of $1 \mathrm{mg} /$ day, gradually increasing to $2.5 \mathrm{mg}$ /day.

Case 2. A 41-year-old man with recurrent exudative pericarditis, associated with orbital, mediastinic, and retroperitoneal fibrosis, treated with methylprednisolone (60 mg/day), indomethacin (50 mg/day), azathioprine ( $50 \mathrm{mg} /$ day), and tamoxifen $(10 \mathrm{mg} /$ day) was admitted to our hospital in 2007. Laboratory testing showed high acute-phase reactant. Colchicine was added to therapy at a dosage of $1 \mathrm{mg} /$ day and all the other drugs were gradually suspended.

Case 3. A 62-year-old man with ankylosing spondylitis with periaortic RPF since 2005 was treated with methylprednisolone (40 mg every 3 weeks) for 2 years with little benefit. Colchicine was started at a dosage of $1 \mathrm{mg} /$ day, avoiding increase of corticosteroids.

Case 4. A 70-year-old man with Behçet's disease since 1979 and periaortic exudative RPF since 2004, treated with high doses of prednisone ( $25 \mathrm{mg} /$ day for 30 years), was admitted in February 2009 for fever and skin lesions with high levels of acute-phase reactant. After colchicine administration at a dosage of $1 \mathrm{mg}$ /day, subsequently increased to $1.5 \mathrm{mg} /$ day, corticosteroids were reduced to $7.5 \mathrm{mg} /$ day.

In the first patient, after 1 year, the colchicine alone, besides preventing acute episodes, allowed complete resolution of RPF (Figure 2). After 4 years, the patient is still asymptomatic.

In the other patients, a clinical and radiological improvement was obtained with disappearance of symptoms and normalized levels of acutephase reactant, and significant (more than 60\%) regression of RPF, as shown by radiological examinations. After 3 years for the second and third patient and after 1 year for fourth, no disease recurrence/progression occurred.

We describe 4 cases in which colchicine was effective not only to prevent acute attacks of the autoinflammatory diseases, but also to induce remission of RPF. Although the incidence is low, estimated at fewer than 1

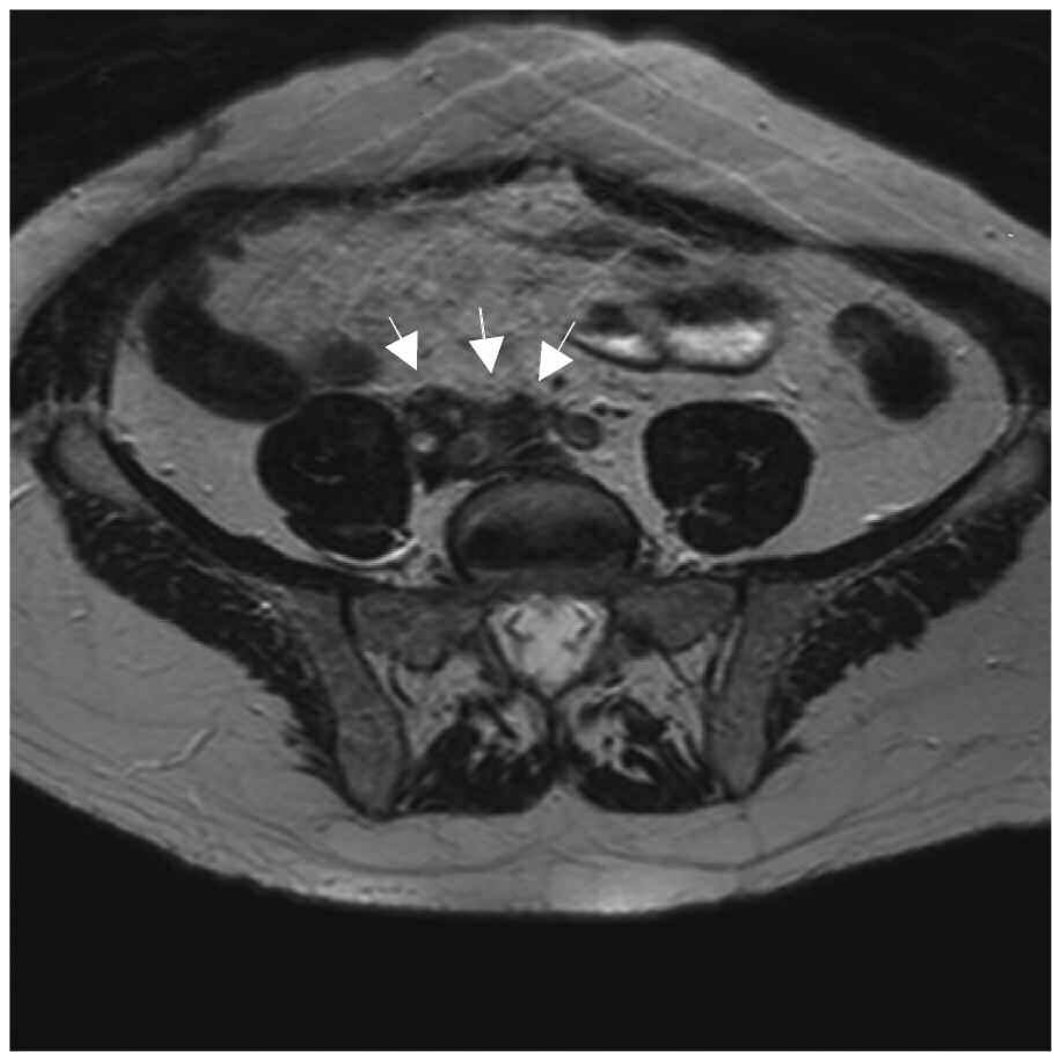

Figure 1. Magnetic resonance imaging in 2004 of periaortic retroperitoneal fibrosis in Patient 1. Arrows indicate the extent of fibrosis. 


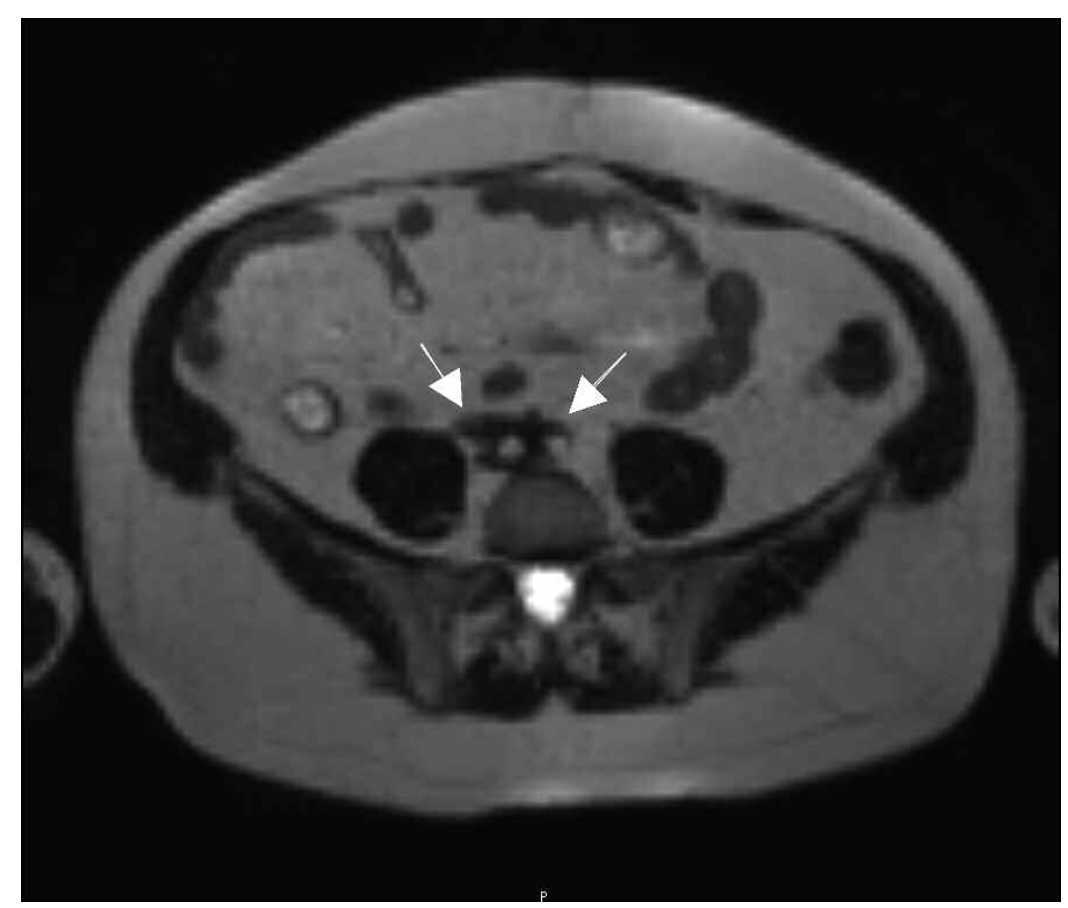

Figure 2. Magnetic resonance imaging in 2007 showing complete resolution of retroperitoneal fibrosis. Arrows indicate the extent of fibrosis.

event/100,000 population ${ }^{6}$, RPF is an increasingly recognized syndrome. Nevertheless there are no guidelines or controlled therapeutic trials for the treatment. RPF rarely remits spontaneously, and prolonged therapy usually is required. Steroids at high doses and tamoxifen are the drugs most used; other agents such as azathioprine, methotrexate, mycophenolate, cyclophosphamide, and cyclosporine are usually given to nonresponders $^{7,8}$. New evidence about colchicine's effectiveness in RPF and other fibrosing diseases is emerging. Recently the efficacious use of colchicine associated with corticosteroids in the treatment and prevention of recurrence of RPF was reported ${ }^{8,9}$.

We described the first case of remission of RPF after 1 year of treatment with colchicine alone ${ }^{10}$. The result was confirmed after 4 years of followup.

In the cases described here, corticosteroids, tamoxifen, and cyclophosphamide were shown to be ineffective. Colchicine alone or its addition to the therapy with concomitant reduction of corticosteroids was effective not only to induce complete or significant remission of RPF, but also to prevent disease recurrence.

The coincidental association of RPF with autoinflammatory diseases allowed us to observe the benefit of colchicine. Colchicine should be considered as a therapeutic tool in the treatment of RPF in future prospective and comparative trials.

GIULIANA de SOCIO, MD; ELENA VERRECCHIA, MD; CLAUDIA FONNESU, MD; MARIA GIOVINALE, MD; GIOVANNI BATTISTA GASBARRINI, MD, PhD; RAFFAELE MANNA, MD, PhD, Department of Internal Medicine, Catholic University, Rome, Italy.

Address correspondence to Dr. R. Manna, Department of Internal Medicine, Catholic University, largo A. Gemelli 8, 00168 Rome, Italy. E-mail: rmanna@rm.unicatt.it

\section{REFERENCES}

1. Burkhardt Soares S, Fehr A, Brandt AS, Roth S. Retroperitoneal fibrosis. Aktuelle Urol 2007;38:221-31.

2. Douglas WW, Ryu JH, Schroeder DR. Idiopathic pulmonary fibrosis: Impact of oxygen and colchicine, prednisone, or no therapy on survival. Am J Respir Crit Care Med 2000;161(4 Pt 1):1172-8.

3. Davies HR, Richeldi L. Idiopathic pulmonary fibrosis: current and future treatment options. Am J Respir Med 2002;1:211-24.

4. Bhat A, Naguwa SM, Cheema GS, Gershwin ME. Colchicine revisited. Ann NY Acad Sci 2009;1173:766-73.

5. Tzortzaki EG, Antoniou KM, Zervou MI, Lambiri I, Koutsopoulos A, Tzanakis N, et al. Effects of antifibrotic agents on TGF-beta 1, CTGF and IFN-gamma expression in patients with idiopathic pulmonary fibrosis. Respir Med 2007;101:1821-9.

6. Vaglio A, Salvarani C, Buzio C. Retroperitoneal fibrosis. Lancet 2006;367:241-51

7. Scheel PJ, Piccini J, Rahman MH, Lawler L, Jarrett T. Combined prednisone and mycophenolate mofetil treatment for retroperitoneal fibrosis. J Urol 2007;178:140-3.

8. Swartz RD. Idiopathic retroperitoneal fibrosis: a review of the pathogenesis and approaches to treatment. Am J Kidney Dis 2009;54:546-53.

9. Vega J, Goecke H, Tapia H, Labarca E, Santamarina M, Martinez G. Treatment of idiopathic retroperitoneal fibrosis with colchicine and steroids: a case series. Am J Kidney Dis 2009;53:628-37.

10. de Socio G, Cerquaglia C, Curigliano V, Fonnesu C, Giovinale M, Verrecchia E, et al. Association between familial Mediterranean fever and retroperitoneal fibrosis: retroperitoneal fibrosis regression after colchicine therapy. Int J Immunopathol Pharmacol 2009;22:521-4.

J Rheumatol 2010;37:9; doi:10.3899/jrheum.100352 